\title{
GPR160 de-orphanization reveals critical roles in neuropathic pain in rodents
}

\author{
Gina L.C. Yosten, ${ }^{1,2}$ Caron M. Harada, ${ }^{1,2}$ Chris Haddock, ${ }^{1,2}$ Luigino Antonio Giancotti, ${ }^{1,2}$ Grant R. Kolar, ${ }^{2,3}$ Ryan Patel, ${ }^{4}$ Chun Guo, \\ Zhoumou Chen, ${ }^{1,2}$ Jinsong Zhang, ${ }^{1,2}$ Timothy M. Doyle, ${ }^{1,2}$ Anthony H. Dickenson, ${ }^{4}$ Willis K. Samson, ${ }^{1,2}$ and Daniela Salvemini ${ }^{1,2}$ \\ 'Department of Pharmacology and Physiology, ${ }^{2}$ Henry and Amelia Nasrallah Center for Neuroscience, and ${ }^{3}$ Department of Pathology, Saint Louis University School of Medicine, St. Louis, Missouri, USA. \\ ${ }^{4}$ Neuroscience, Physiology and Pharmacology, University College London, London, United Kingdom.
}

\begin{abstract}
Treating neuropathic pain is challenging and novel non-opioid-based medicines are needed. Using unbiased receptomics, transcriptomic analyses, immunofluorescence, and in situ hybridization, we found that the expression of the orphan GPCR Gpr160 and GPR160 increased in the rodent dorsal horn of the spinal cord following traumatic nerve injury. Cenetic and immunopharmacological approaches demonstrated that GPR160 inhibition in the spinal cord prevented and reversed neuropathic pain in male and female rodents without altering normal pain response. CPR160 inhibition in the spinal cord attenuated sensory processing in the thalamus, a key relay in the sensory discriminative pathways of pain. We also identified cocaine- and amphetamine-regulated transcript peptide (CARTp) as a GPR160 ligand. Inhibiting endogenous CARTp signaling in spinal cord attenuated neuropathic pain, whereas exogenous intrathecal CARTp evoked painful hypersensitivity through GPR160-dependent ERK and CAMP response element-binding protein (CREB). Our findings de-orphanize GPR160, identify it as a determinant of neuropathic pain and potential therapeutic target, and provide insights into its signaling pathways. CARTp is involved in many diseases including depression and reward and addiction; de-orphanization of GPR160 is a major step forward understanding the role of CARTp signaling in health and disease.
\end{abstract}

\section{Introduction}

Neuropathic pain conditions arising from injuries to the nervous system due to trauma, disease, or neurotoxins are chronic, severe, debilitating, and exceedingly difficult to treat (1). Opioids are widely used to treat chronic pain but limited by severe side effects and strong abuse liability (2). Neuropathic pain is a burgeoning global medical issue (e.g., $>15$ million people in the US and $>20 \%$ of the European population; refs. 3, 4) with a profound annual economic burden of treatment (5). When combined with over 15 million people worldwide having experienced an opioid-use disorder (6), a high priority has been placed on developing novel non-opioid-based analgesics.

GPCRs are the most abundant receptor family and regulate a diverse array of cellular functions, including neurotransmission in pain (7). Approximately 120 of 400 nonsensory GPCRs are considered orphan GPCRs (oGPCRs), as their cognate ligands are unknown (8). Using a multidisciplinary approach, we present the first evidence to our knowledge that the oGPCR GPR160 in the spinal cord plays critical roles in the development and maintenance of hypersensitivity associated with traumatic nerve injury-induced neuropathic pain. We also identify cocaineand amphetamine-regulated transcript peptide (CARTp) (9) as a GPR160 ligand and unraveled signaling pathways engaged

Conflict of interest: DS, GLCY, and WKS have a patent on the discovery of antagonists of GPR160 for the treatment of pain (W02017011738).

Copyright: @ 2020, American Society for Clinical Investigation.

Submitted: September 5, 2019; Accepted: January 23, 2020; Published: April 13, 2020

Reference information: J Clin Invest. 2020;130(5):2587-2592.

https://doi.org/10.1172/JCI133270. downstream of GPR160. These findings provide the foundation for investigating GPR160 as a potential therapeutic target for treating chronic pain.

\section{Results and Discussion}

GPR160 is upregulated in the spinal cord during neuropathic pain. Our receptomic approach (10) (Supplemental Figure 1A; supplemental material available online with this article; https://doi. org/10.1172/JCI133270DS1) identified 4 main clusters (Supplemental Figure 1B) among non-orphan GPCRs known to be altered in neuropathic pain states $(11,12)$. Querying the sequence homology of oGPCRs against GPCRs in these clusters identified 31 candidate oGPCRs (Supplemental Figure 1C). We surveyed their expression in the spinal cord from rats with chronic constriction of the sciatic nerve-induced (CCI-induced) neuropathic pain, which produces mechanohypersensitivities that peak by day 7 and last for several weeks (13). PCR and quantitative PCR analyses of these oGPCRs revealed that Gpr160 significantly increased in the dorsal, but not the ventral, horn of the spinal cord ipsilateral to injury (Figure 1A). As a control, Gpr107, which is expressed in the dorsal horn of the spinal cord (DH-SC) but not homologous to any of our branch clusters, was not significantly changed by CCI (Figure 1A). No significant changes in Gpr160 were observed in the dorsal root ganglia $(n=3 ; P=0.5)$.

RNA sequencing (RNA-Seq) analyses of ipsilateral rat DH-SC following CCI or sham injury identified 60 differentially expressed GPCRs between CCI and sham groups. Remarkably, Gpr160 was one of the $G p r$ transcripts with the greatest differential expression $\left(4.44\right.$-fold change; false discovery rate $\left.=6.06 \times 10^{-12}\right)$ in the CCI group (Figure 1, B and C). 
A

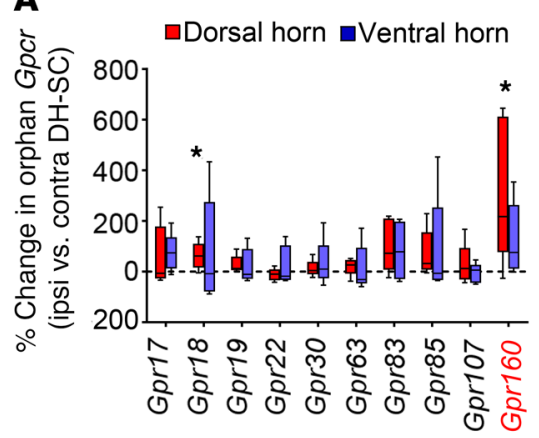

B

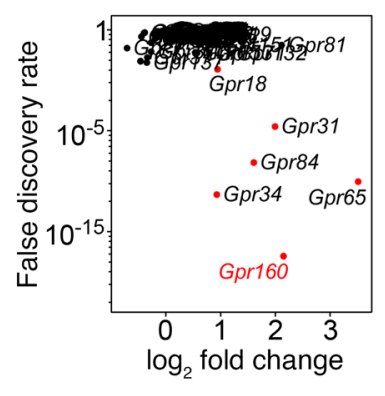

C

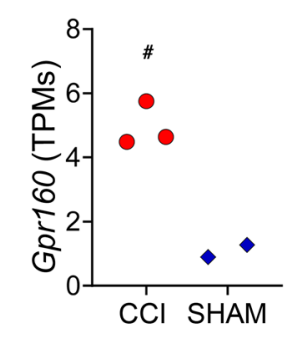

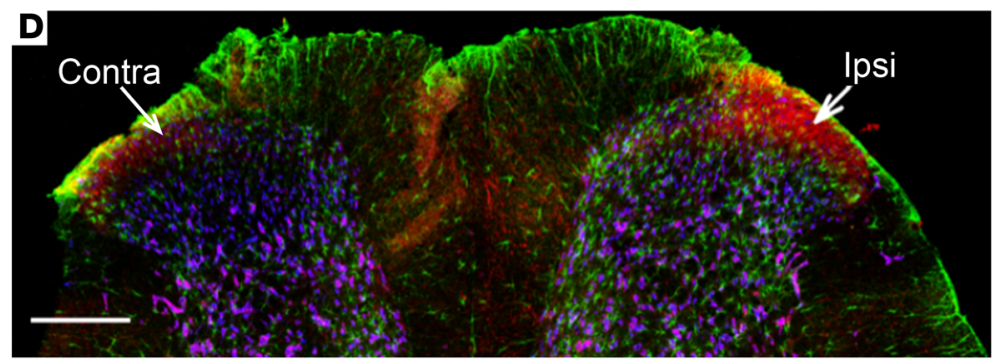

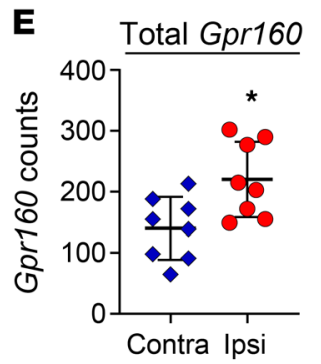

$\mathbf{F}$

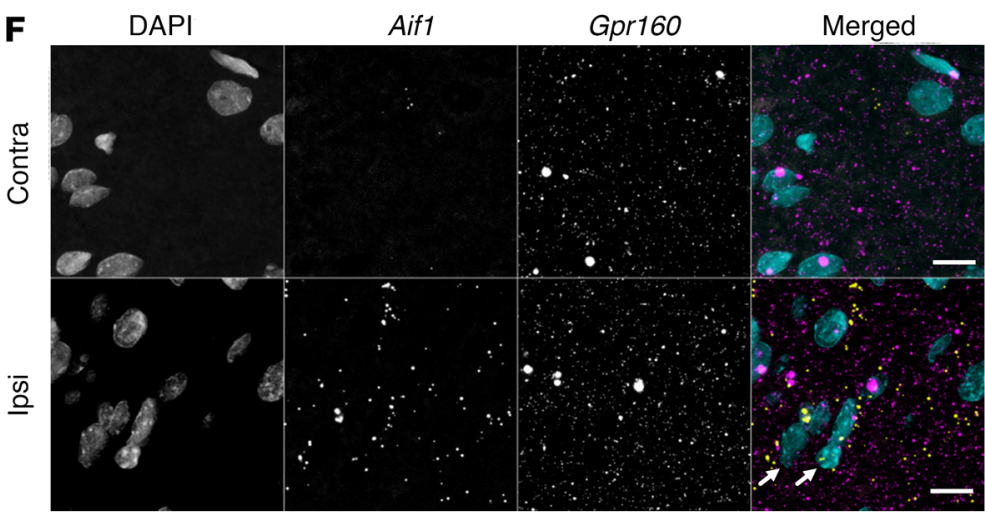

G

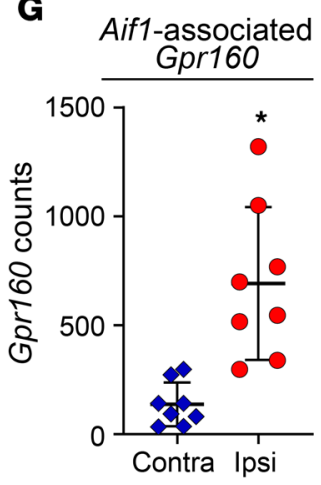

Figure 1. Gpr160 and GPR160 upregulation in the spinal cord following CCI. (A) Quantitative real-time PCR analysis of oCPCR mRNA expression in the dorsal and ventral horns of the spinal cord from rats with $\mathrm{CCI}$ on day $7(n=5)$. (B and $\mathbf{C})$ RNA-Seq analyses of rat DH-SC ipsilateral to CCI on day 9. (B) Differential expression of 60 GPCRs between CCI and sham ( $n=3 /$ group). (C) Gpr160 in CCI and SHAM. TPMs, total reads per million. (D) Immunolabeled GPR160 (red) in lamina I/II spinal cord of rats with CCI. Ipsilateral (Ipsi), contralateral (Contra), GFAP (green), and NeuN (blue). (E-G) RNAScope analyses of the rat DH-SC on day 10 after CCI. (E) Quantitation of total Gpr160. (F and G) Association (white arrows; F) of Gpr160 (magenta) and Aif1 (microglia; yellow) increased ipsilateral to CCI (G). Nuclei were stained with DAPI (cyan). Scale bars: $100 \mu \mathrm{m}$ (D) or $10 \mu \mathrm{m}$ (F). Data are expressed as (A) median, interquartile range, and minimum/maximum values or (E and $\mathbf{G}$ ) mean \pm SD. (A-C, E, and $\mathbf{C})$ Data analyzed by 2-tailed Student's $t$ test; (B and $\mathbf{C}$ ) adjusted by BenjaminiHochberg false discovery rate. ${ }^{*} P<0.05$ versus Contra and ${ }^{\sharp} P<0.05$ and $q<0.05$ versus sham.

GPR160 is highly conserved among species and expressed on neurons, astrocytes, and microglia $(14,15)$ in human and rodent CNS, including the spinal cord $(15,16)$. Image analyses of spinal cord from rats with CCI revealed increased GPR160 (26.9\% \pm $5.6 \%$ SEM, $n=5 /$ group, $P=0.042$, paired $t$ test; Figure 1D) and Gpr160 (Figure 1E) within lamina I and II of ipsilateral DH-SC compared with the contralateral side. When measured in lamina I and II of the DH-SC (Supplemental Figure 3A), Gpr160 was expressed in astrocytes (Supplemental Figure 2A and Supplemental Figure 3B), microglia (Figure $1 \mathrm{~F}$ and Supplemental Figure 3C), and neurons (Supplemental Figure $2 \mathrm{C}$ and Supplemental Figure 3D). However, Gpr160 was significantly increased in proximity to Aifl (Figure 1G), but not Gfap (Supplemental Figure 2B) or Rbfox3 (Supplemental Figure 2D), suggesting microglia may account for CCI-induced Gpr160 and GPR160 expression in the spinal cord.
Activation of GPR160 in the spinal cord contributes to neuropathic pain. The functional contribution of GPR160 at this site was tested using genetic (siRNA) and immunopharmacological (neutralizing antibody $[\mathrm{Ab}]$ ) approaches, since there are no smallmolecule GPR160 antagonists. Daily intrathecal (i.th.) injections of siGpr160, but not control siRNA (sieGfp), blocked mechanoallodynia in the rat CCI model (Figure 2A) and a second rat model of traumatic nerve injury (spared nerve injury; ref. 17) (Figure 2B). Moreover, i.th. siGpr160 at a time of peak CCI-induced mechano-allodynia (day 7 and 8) significantly reversed allodynia (Figure 2C) and reduced Gpr160 in the ipsilateral DH-SC by approximately $40 \%(n=5)$. Injection (i.th.) of neutralizing anti-GPR160 Ab at a time of peak neuropathic pain (day 8) also reversed mechanoand cold-allodynia in male rats by 30 minutes, with peak reversal effects by 2 hours (Figure 2, D and E) and resolution by 6 hours. 
A

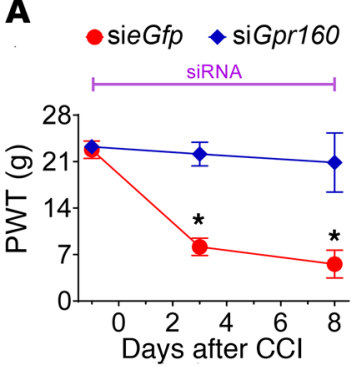

B

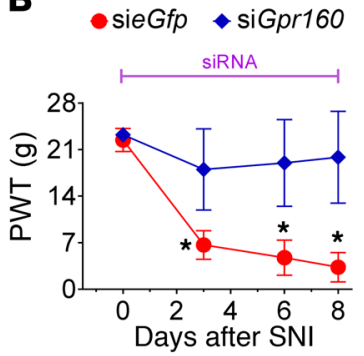

C

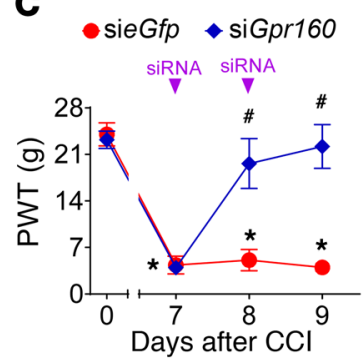

D
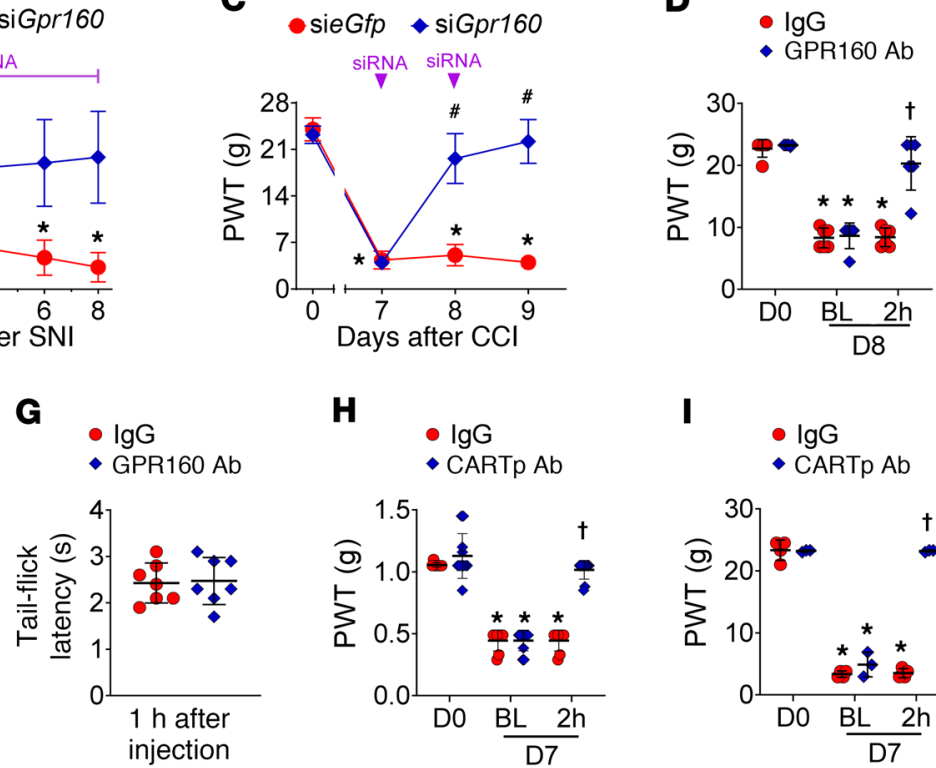

H

- $\lg G$ - cartp ab

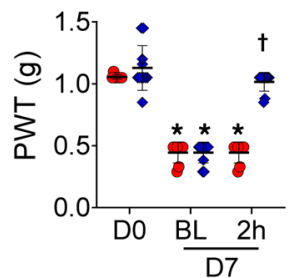

I

- IgG

- CARTp Ab

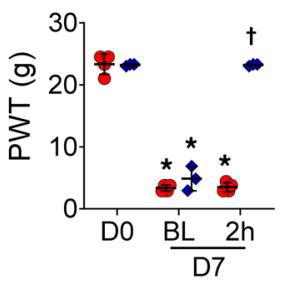

$\mathbf{J}$

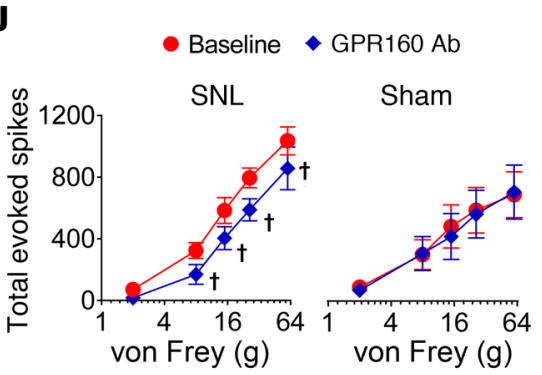

$\mathbf{K}$

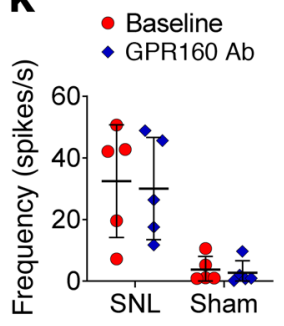

$\mathbf{L}$

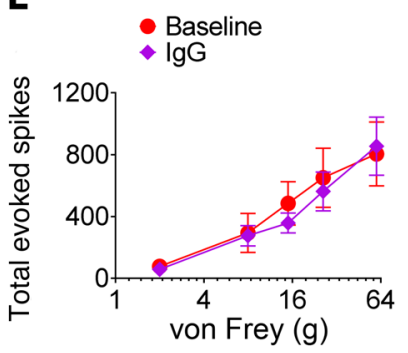

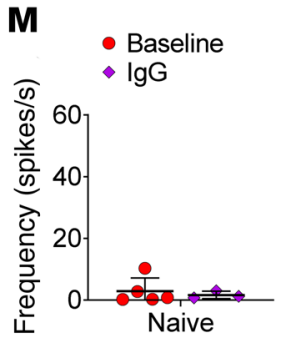

Figure 2. GPR160 inhibition attenuated and reversed neuropathic pain. (A) CCI-induced and (B) spared nerve injury-induced (SNI-induced) mechanoallodynia in male rats were prevented by daily i.th. $\operatorname{siCpr160}(\mathbf{A}, n=6 ; \mathbf{B}, n=4)$, but not sieGfp control $(\mathbf{A}, n=7 ; \mathbf{B}, n=4)$. (C) CCI-induced mechano-allodynia in male rats was reversed by i.th. siGpr160, but not sieGfp ( $n=3$ /group). CCI-induced (D) mechano-allodynia ( $n=6 /$ group) and (E) cold-allodynia ( $n=3$ / group) in male rats was reversed with i.th. GPR160 Ab, but not with nonspecific IgG. (F) Intrathecal GPR160 Ab $(n=4)$, but not IgG $(n=5)$, reversed CCIinduced mechano-allodynia in female rats. (C) Intrathecal GPR160 Ab or IgG ( $n=7 /$ group) in normal male rats had no effect on tail-flick nociceptive responses. (H and I) Intrathecal CARTp Ab $(\mathbf{H}, n=11 ; \mathbf{I}, n=3)$, but not IgG $(\mathbf{H}, n=8 ; \mathbf{I}, n=4)$, reversed CCI-induced mechano-allodynia in male mice (H) and rats (I). When compared with baseline, i.th. GPR160 Ab $(n=5)$ attenuated neuronal responses to punctate mechanical stimuli $(\mathrm{J})$, but not ongoing neuronal activity (K) in spinal nerve ligation (SNL), but not sham, rats. No effects on neuronal responses to punctate mechanical stimuli (L) or ongoing neuronal activity $(\mathbf{M})$ were observed with IgC $(n=4)$ in naive rats. Data are expressed as mean \pm SD $(\mathbf{A}-\mathbf{I})$ or mean \pm SEM $(\mathbf{J}-\mathbf{M})$ and analyzed by 2-tailed, 2-way repeated-measures ANOVA with Bonferroni's multiple-comparisons test (A-F and $\mathbf{H}-\mathbf{L}$ ) or 2 -tailed $t$ test (G and $\mathbf{M})$. ${ }^{*} P<0.05$ versus day 0 (D0), ${ }^{\#} P<0.05$ versus $\mathrm{D7}$, and ${ }^{\dagger} P<0.05$ versus baseline (BL). PWT, paw withdrawal threshold.

Similar results were obtained in female rats (Figure 2F). GPR160 inhibition did not produce observable adverse health effects or alter normal nociceptive thresholds (tail-flick latency; ref. 18) in noninjured rats (Figure 2G). These results suggest selective alleviation of chronic pain states without impact on beneficial and protective nociceptive responses.

We examined the effect of anti-GPR160 Ab on neuronal processing within the spinothalamic-ventrobasal-somatosensory cortical pathway to peripherally applied sensory modalities using in vivo electrophysiological recordings from the ventral posterolateral thalamus (Supplemental Figure 4A), a key relay in the sensory discriminative pathways of pain in the brain. Baseline evoked and ongoing neuronal activities were comparable to our previous observations (19). GPR160 Ab administered i.th. produced modalityselective inhibitory effects similar to pregabalin (19) in rats with spinal nerve ligation that were dependent on the pathophysiological state, similar to behavior and demonstrative of sensory process- ing rather than motor responses. When compared with baseline, the neuronal responses to punctate mechanical stimuli following GPR160 Ab were reduced to a range of low intensity von Frey filaments ( 2 and $8 \mathrm{~g}$ ) and those likely to exceed withdrawal thresholds $(>15 \mathrm{~g})$, whereas there were no changes observed in sham groups (Figure 2J and Supplemental Figure 4B). No inhibitory effects were observed on evoked neuronal responses to heat (Supplemental Figure 4C), innocuous (Supplemental Figure 4D) and noxious evaporative cooling (Supplemental Figure 4E), or brush stimulation of the receptive field (Supplemental Figure 4F) in either group. Ongoing neuronal activity was also unaltered after dosing in either group (Figure 2K). A control antibody $(\mathrm{IgG}$ ) in naive rats had no effect on all evoked and spontaneous measures (Figure 2, L and M, and Supplemental Figure 4). These results show that GPR160 in the spinal cord contributes to the ascending transmission of sensory inputs within sensory-discriminative projection pathways. The lack of effect in sham animals and on thermal and 

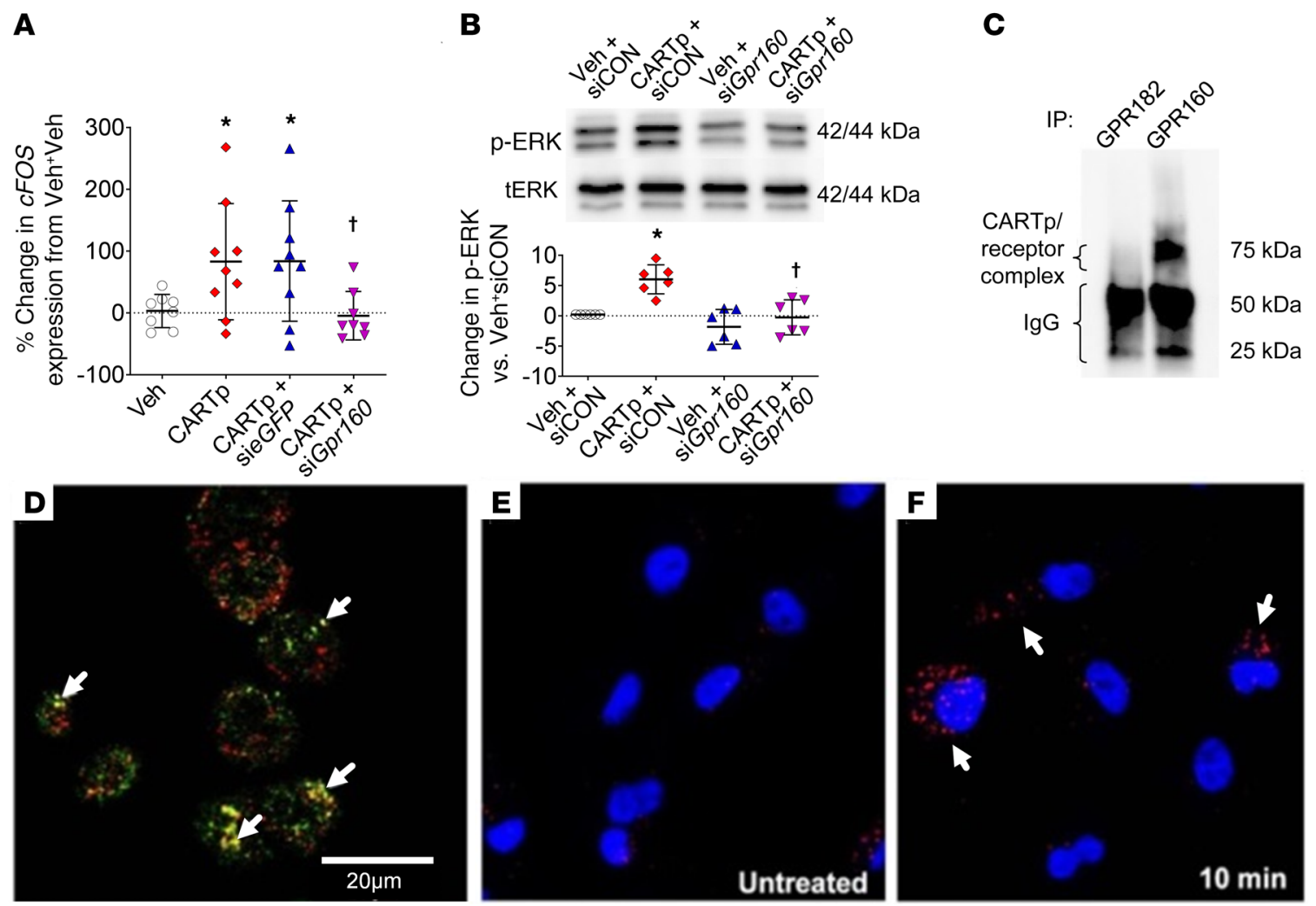

Figure 3. CARTp is a GPR160 ligand. (A and B) CARTp-stimulated (A) cFOS in human KATO III cells and (B) ERK phosphorylation in PC-12 cells; events attenuated with siGpr160, but not control siRNA (A, sieGfp or B, siCON [noncoding scrambled siRNA]; $n=3$ experiments/group with 3 replicates/experiment). tERK, total ERK. (C) In KATO III cell lysates, exogenous CARTp coimmunoprecipitated with GPR160 ( $n=3$ experiments/group with 3 replicates/ experiment). (D) FAM-labeled CARTp (green) colocalized (yellow; white arrows) with GPR160 (red) in KATO III cells ( $n=3$ experiments with 1 replicate/ experiment). (E and F) Proximity ligation assay revealed the close proximity (red) of CARTp and CPR160 ( $n=3$ experiments with 2 replicates/experiment) in CARTp-treated cells (F), but not in untreated cells $(\mathbf{E})$. Blue = nuclear staining. Data are expressed as mean \pm SD and were analyzed by 2-tailed 1-way ANOVA with Dunnett's multiple-comparisons test. ${ }^{*} P<0.05$ versus Veh and ${ }^{\dagger} P<0.05$ versus CARTp + sieGfp/siCON.

ongoing activity indicates a selective role of GPR160 inhibition in these pathophysiological evoked responses that is similar to pregabalin (19). One discrepancy between the behavioral and neuronal data was the lack of effect in cold responses of the sensory neurons. This may be due to the complexities of cold processing and the suprathreshold nature of the neuronal cold stimulus that might employ different molecular mechanisms between behavioral and electrophysiological tests. Differences between the models used may also contribute. The neuronal responses extend to suprathreshold stimuli and so represent coding of high intensity stimuli that could equate better to high pain scores in patients with neuropathy than threshold responses that can be measured in behavioral studies (20).

CARTP - a ligand of GPR160. Comparing tissue expression profiles (NCBI Gene) of GPR160 and endogenously expressed orphan ligands revealed high correlation between GPR160 and CARTp. CARTp has 2 bioactive forms in rat (CARTp 55-102 and CARTp 62-102; ref. 21) and human (CARTp 42-89 and CARTp 49-89; ref. 21). CARTp 55-102, the most widely used isoform, acts through a $\mathrm{G} \alpha_{\mathrm{i} / 0}$-coupled GPCR linked to the activation (phosphorylation) of ERK $(22,23)$. Using cell culture, we deorphanized GPR160 by identifying a functional and potential physical connection between CARTp and GPR160. In human KATO III cancer cells that express GPR160 (Supplemental Fig- ure 5A), CARTp induced $c F O S$ expression, which was completely blocked in siGpr160-transfected cells (Figure 3A). Rat pheochromocytoma cells (PC-12), which can be differentiated into a neuronal-like phenotype, expressed GPR160 (Supplemental Figure 5, B and C). CARTp stimulated ERK phosphorylation (p-ERK) in PC-12 cells that was attenuated in siGpr160-transfected cells (Figure 3B). The efficacy of siRNA was confirmed by reduced Gpr160 and GPR160 levels (Supplemental Figure 5, B and C). These findings suggest a functional relationship between CARTp and GPR160 and reveal that GPR160 confers CARTp-induced cFOS and phosphorylation of ERK. Moreover, CARTp coimmunoprecipitated with GPR160 (Figure 3C), suggesting a potential physical interaction. The $75-\mathrm{kDa}$ complex is greater than the predicted $52-\mathrm{kDa}$ complex and may reflect additional proteins that associate to form the signalosome. We also found exogenous CARTp colocalized with GPR160 (Figure 3, D-F). We have previously demonstrated that GPR160 does not interact with another peptide hormone, proinsulin C-peptide (24), suggesting the potential CARTp-GPR160 interaction is likely specific.

CARTp is expressed in the CNS, including the superficial laminae of the rat spinal cord $(25,26)$. Cartp is expressed in mouse cerebral cortex glia (microglia and astrocytes) and neurons (14). However, the contribution of CARTp/GPR160 signaling to nociceptive processing is not known. An i.th. injection of a neutralizing CARTp 
A

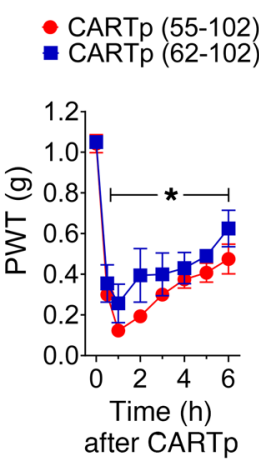

B

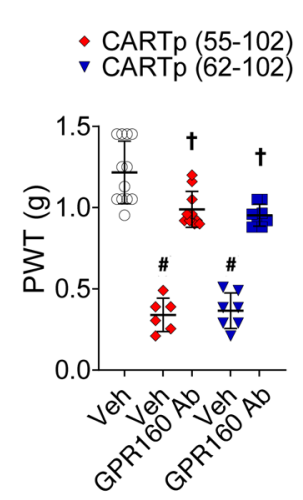

C

- CARTp (55-102)

- CARTP (62-102)

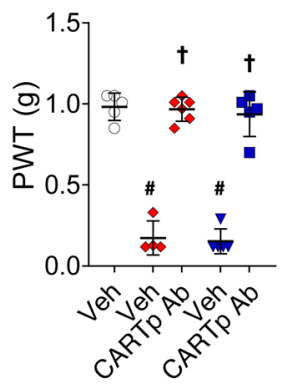

D

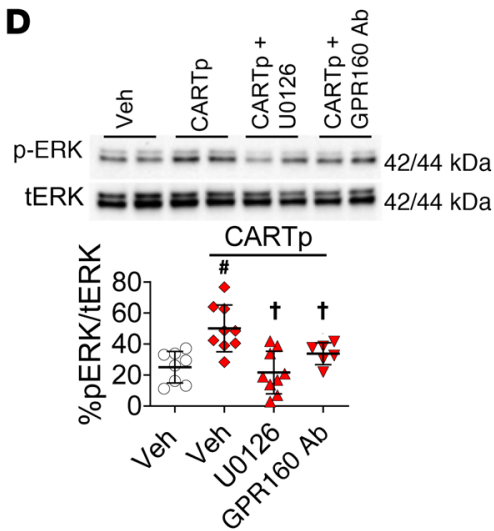

$\mathbf{E}$

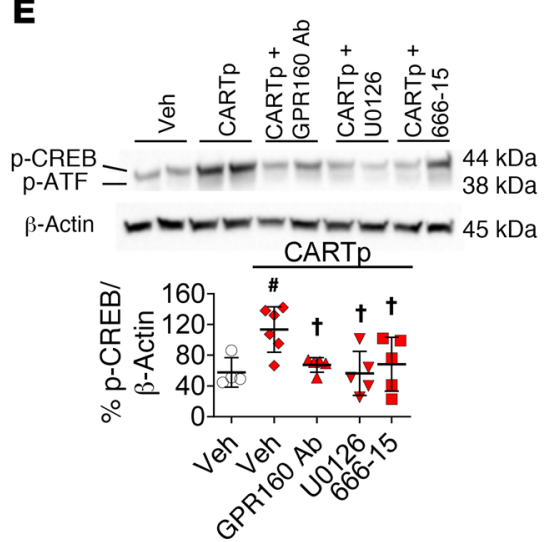

$\mathbf{F}$

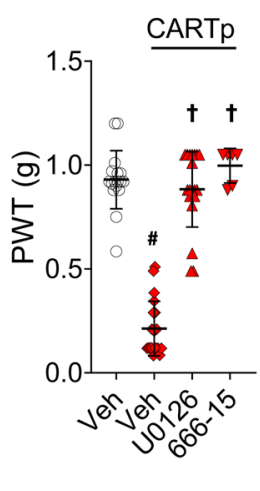

G

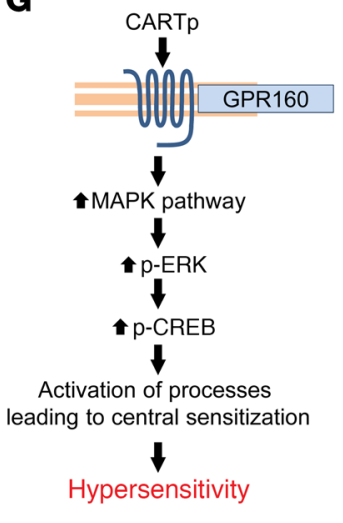

Figure 4. CARTp induced mechano-hypersensitivity in mice through GPR160-dependent ERK-CREB activation in the spinal cord. (A) Time-dependent development of mechano-allodynia in mice $(n=4)$ after i.th. CARTp 55-102 or CARTp 62-102. (B and C) Mechano-allodynia measured 1 hour after i.th. CARTp 55-102 (B, $n=6 ; \mathbf{C}, n=4)$ or CARTp 62-102 (B, $n=7 ; \mathbf{C}, n=5$ ) was reduced with i.th. GPR160 Ab (B, $n=10$ and $n=8$, respectively) or i.th. CARTp Ab (C, $n=6$ and $n=5$, respectively). ( $\mathbf{D}$ and $\mathbf{E}$ ) When compared with vehicle $(\mathbf{D}, n=8 ; \mathbf{E}, n=4)$, i.th. CARTp 55-102 induced phosphorylation of ERK ( $\mathbf{D} ; \mathrm{p}$-ERK, $n=9$ ) and CREB (E; p-CREB, $n=6$ ) in the DH-SC, which was attenuated with i.th. coinjections of MEK inhibitor U0126 (D, $n=9 ; \mathbf{E}, n=5)$, CREB inhibitor 666-15 (E, $n=5)$, or GPR160 Ab (D, $n=6)$. (F) CARTp 55-102-induced mechano-allodynia $(n=20)$ was attenuated with coinjection of U0126 $(n=20)$ or 666-15 $(n=6)$. Vehicles for CARTp, U0126, and 666-15 ( $n=17)$ had no effect on behavior. (C) Proposed model of CARTp/GPR160-induced signaling. Data are expressed as mean \pm SD and were analyzed by (A) 2-tailed, 2-way ANOVA with Bonferroni's multiple-comparisons test or (B-F) 2-tailed, 1-way ANOVA with Dunnett's multiple-comparisons test. ${ }^{*} P<0.05$ versus 0 hours; ${ }^{\#} P<0.05$ versus Veh; and ${ }^{\dagger} P<0.05$ versus respective CARTp plus Veh. PWT, paw withdrawal threshold.

$\mathrm{Ab}$ at a time of peak $\mathrm{CCI}-$-induced neuropathic pain reversed mechano-allodynia in mice and rats in a time-dependent fashion by 30 minutes, with peak reversal by 2 hours (Figure 2, $\mathrm{H}$ and I) and resolution within 5 to 6 hours. The effects of CARTp Ab mimicked those noted with GPR160 inhibition (Figure 2, D and E), suggesting that CARTp/GPR160 signaling occurs in response to nerve injury.

CARTp induces GPR160-mediated hypersensitivities through ERK/CREB signaling in the spinal cord. If CARTp/GPR160 in the spinal cord following nerve injury contributes to the development of neuropathic pain, then i.th. injections of CARTp should recapitulate behavioral consequences of neuropathic pain states. Results from early studies of CARTp in pain were inconclusive due in part to limited data and contradictory pro- and antinociceptive effects in the CNS $(25,27,28)$. Reported antinociceptive effects of CARTp manifested at very high doses of the peptide $(\mu \mathrm{g})$ and the purity of the peptide preparation was not known or not reported $(27,28)$. In contrast, Ohsawa and colleagues reported that i.th. injections of low dose (3-100 ng), highly purified (>96\%) CARTp induced thermal hyperalgesia in a dose-dependent fashion (25). Using the same purity of CARTp and supplier as Ohsawa, a single i.th. injection of CARTp (3-30 ng) in mice caused profound mech- ano-allodynia in pilot studies that peaked by 1 hour and persisted over 5 hours. As shown in Figure 4A, CARTp (30 ng) caused near-to-maximal allodynia by 1 hour that was abolished by i.th. injection of GPR160 Ab (Figure 4, A and B) or CARTp Ab (Figure 4C), providing support that CARTp-induced mechanohypersensitivity is dependent on GPR160.

CARTp-induced ERK signaling stimulates the phosphorylation and activation of CREB independently of cAMP signaling (22). ERK can serve as an upstream regulator of CREB phosphorylation (p-CREB) (29) during the development of neuropathic pain (30). We found i.th. CARTp induced GPR160-mediated ERK/CREB signaling in the mouse DH-SC that contributed to the development of mechano-allodynia. First, i.th. CARTp induced the phosphorylation of ERK (Figure 4D) and CREB (Figure 4E), which was attenuated by coadministration of GPR160 Ab (Figure 4, D and E). Secondly, inhibiting MAPK/ERK kinases (MEK 1 and 2) with a MEK1/2 inhibitor, U0126 (31), attenuated CARTp-induced mechano-allodynia (Figure 4F) and phosphorylation of ERK (Figure 4D) and CREB (Figure 4E). Finally, CARTp-induced mechano-allodynia (Figure $4 \mathrm{~F}$ ) and $\mathrm{CREB}$ phosphorylation (Figure $4 \mathrm{E}$ ) were attenuated by i.th. administration of the CREB inhibitor, 666-15 (32). 
Using an array of multidisciplinary approaches, we found a role for CARTp/GPR160 signaling (Figure 4G) in spinal cord in neuropathic pain. These findings provide the kernel for future investigation of GPR160 signaling in pain and other CARTp-associated diseases, including anxiety and depression, reward and addiction, and food intake and maintenance of body weight (21). Accordingly, our findings set the stage for medicinal discovery efforts to identify small-molecule antagonists of GPR160 for the treatment of neuropathic pain with broader implication for the treatment of additional disease states.

\section{Methods}

Detailed methods are provided in the supplemental materials.

Study approval. All experiments were performed in accordance with the International Association for the Study of Pain, the NIH guidelines on laboratory animal welfare, The Animals (Scientific Procedures) Act 1986/directive 2010/63/EU, and approved by the Saint Louis University Institutional Animal Care, internal ethics committee at the University College London, and the UK Home Office.
RNA-Seq data are available through the NCBI's Gene Expression Omnibus (GEO) repository (GSE143895).

\section{Author contributions}

GLCY, CMH, CH, LAG, GRK, CG, ZC, JZ, TMD, RP, and AHD performed experiments and data analyses. CMH, LAG, GRK, JZ, TMD, RP, and AHD assisted in writing the manuscript. GLCY, WKS, and DS conceived experiments and wrote the manuscript.

\section{Acknowledgments}

We thank Andrew Lechner (Saint Louis University School of Medicine) for editorial input and the Saint Louis University Research Microscopy and Histology Core for their contributions. This study was funded by startup funds, the Presidents Research Fund (Saint Louis University), NIH/NINDS grant R01NS1132257 (to DS and GLCY), and NIH/NIDDK grant 1R01DK118340 (to GLCY).

Address correspondence to: Daniela Salvemini, 1402 South Grand Boulevard, St. Louis, Missouri 63104, USA. Phone: 31.977.6430; Email: daniela.salvemini@health.slu.edu.
1. Finnerup NB, et al. Pharmacotherapy for neuropathic pain in adults: a systematic review and meta-analysis. Lancet Neurol. 2015;14(2):162-173.

2. Toblin RL, Mack KA, Perveen G, Paulozzi LJ. A population-based survey of chronic pain and its treatment with prescription drugs. Pain. 2011;152(6):1249-1255.

3. DiBonaventura MD, et al. The prevalence of probable neuropathic pain in the US: results from a multimodal general-population health survey. J Pain Res. 2017;10:2525-2538.

4. Reid KJ, et al. Epidemiology of chronic noncancer pain in Europe: narrative review of prevalence, pain treatments and pain impact. Curr Med Res Opin. 2011;27(2):449-462.

5. Institute of Medicine. Relieving Pain in America: A Blueprint for Transforming Prevention, Care, Education, and Research. Washington, DC: National Academies Press; 2011.

6. Degenhardt L, et al. The global epidemiology and burden of opioid dependence: results from the global burden of disease 2010 study. Addiction. 2014;109(8):1320-1333.

7. Geppetti P, Veldhuis NA, Lieu T, Bunnett NW. G protein-coupled receptors: dynamic machines for signaling pain and itch. Neuron. 2015;88(4):635-649.

8. Alexander SP, et al. The Concise Guide to PHARMACOLOGY 2013/14: G protein-coupled receptors. Br J Pharmacol. 2013;170(8):1459-1581.

9. Zhang M, Han L, Xu Y. Roles of cocaine- and amphetamine-regulated transcript in the central nervous system. Clin Exp Pharmacol Physiol. 2012;39(6):586-592.

10. Yosten GL, Redlinger LJ, Samson WK. Evidence for an interaction of neuronostatin with the orphan G protein-coupled receptor, GPR107. Am JPhysiol Regul Integr Comp Physiol. 2012;303(9):R941-R949.

11. Costigan $\mathrm{M}$, et al. Multiple chronic pain states are associated with a common amino acid-changing allele in KCNS1. Brain. 2010;133(9):2519-2527.

12. $\mathrm{Ma} \mathrm{CH}$, et al. The BMP coreceptor RGMb promotes while the endogenous BMP antagonist noggin reduces neurite outgrowth and peripheral nerve regeneration by modulating BMP signaling. J Neurosci. 2011;31(50):18391-18400.

13. Bennett GJ, Xie YK. A peripheral mononeuropathy in rat that produces disorders of pain sensation like those seen in man. Pain. 1988;33(1):87-107.

14. Zhang Y, et al. An RNA-sequencing transcriptome and splicing database of glia, neurons, and vascular cells of the cerebral cortex. J Neurosci. 2014;34(36):11929-11947.

15. Sathyamurthy A, et al. Massively parallel single nucleus transcriptional profiling defines spinal cord neurons and their activity during behavior. Cell Rep. 2018;22(8):2216-2225.

16. Uhlén M, et al. Proteomics. Tissue-based map of the human proteome. Science. 2015;347(6220):1260419.

17. Decosterd I, Woolf CJ. Spared nerve injury: an animal model of persistent peripheral neuropathic pain. Pain. 2000;87(2):149-158.

18. D'Amour FE, Smith DL. A method for determining loss of pain sensation.J Pharmacol Exp Ther. 1941;72(1):74-79.

19. Patel R, Dickenson AH. Neuronal hyperexcitability in the ventral posterior thalamus of neuropathic rats: modality selective effects of pregabalin. J Neurophysiol. 2016;116(1):159-170.

20. Sikandar S, Dickenson AH. II. No need for translation when the same language is spoken. $\mathrm{Br} J$ Anaesth. 2013;111(1):3-6.

21. Rogge G, Jones D, Hubert GW, Lin Y, Kuhar MJ. CART peptides: regulators of body weight, reward and other functions. Nat Rev Neurosci. 2008;9(10):747-758.

22. Somalwar AR, et al. Cocaine- and amphetamine-regulated transcript peptide (CART) induced reward behavior is mediated via $G_{i / o}$ dependent phosphorylation of PKA/ERK/CREB pathway. Behav Brain Res. 2018;348:9-21.

23. Lakatos A, Prinster S, Vicentic A, Hall RA, Kuhar MJ. Cocaine- and amphetamine-regulated transcript (CART) peptide activates the extracellular signal-regulated kinase (ERK) pathway in AtT20 cells via putative $\mathrm{G}$-protein coupled receptors. Neurosci Lett. 2005;384(1-2):198-202.

24. Yosten GL, Kolar GR, Redlinger LJ, Samson WK. Evidence for an interaction between proinsulin C-peptide and GPR146. J Endocrinol. 2013;218(2):B1-B8.

25. Ohsawa M, Dun SL, Tseng LF, Chang J, Dun NJ. Decrease of hindpaw withdrawal latency by cocaine- and amphetamine-regulated transcript peptide to the mouse spinal cord. Eur J Pharmacol. 2000;399(2-3):165-169.

26. Koylu EO, Couceyro PR, Lambert PD, Kuhar MJ. Cocaine- and amphetamine-regulated transcript peptide immunohistochemical localization in the rat brain. JComp Neurol. 1998;391(1):115-132.

27. Damaj MI, Zheng J, Martin BR, Kuhar MJ. Intrathecal CART (55-102) attenuates hyperlagesia and allodynia in a mouse model of neuropathic but not inflammatory pain. Peptides. 2006;27(8):2019-2023.

28. Upadhya MA, Dandekar MP, Kokare DM, Singru PS, Subhedar NK. Evidence for the participation of cocaine- and amphetamine-regulated transcript peptide (CART) in the fluoxetine-induced anti-hyperalgesia in neuropathic rats. Peptides. 2011;32(2):317-326.

29. Brami-Cherrier K, Roze E, Girault JA, Betuing $\mathrm{S}$, Caboche J. Role of the ERK/MSK1 signalling pathway in chromatin remodelling and brain responses to drugs of abuse. J Neurochem. 2009;108(6):1323-1335.

30. Song XS, Cao JL, Xu YB, He JH, Zhang LC, Zeng YM. Activation of ERK/CREB pathway in spinal cord contributes to chronic constrictive injuryinduced neuropathic pain in rats. Acta Pharmacol Sin. 2005;26(7):789-798.

31. Favata MF, et al. Identification of a novel inhibitor of mitogen-activated protein kinase kinase. J Biol Chem. 1998;273(29):18623-18632.

32. Li BX, et al. Systemic Inhibition of CREB is well-tolerated in vivo. Sci Rep. 2016;6:34513. 